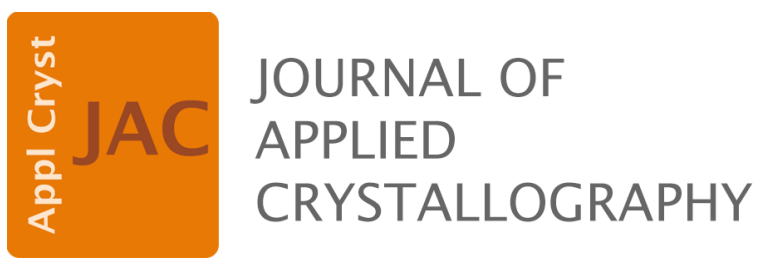

Volume 48 (2015)

Supporting information for article:

Removing multiple outliers and single-crystal artefacts from X-ray diffraction computed tomography data

Antonios Vamvakeros, Simon D. M. Jacques, Marco Di Michiel, Vesna Middelkoop, Christopher K. Egan, Robert J. Cernik and Andrew M. Beale 


\title{
Removing multiple outliers and single crystal artefacts from X-ray diffraction computed tomography data
}

Authors

\author{
Antonios Vamvakeros $^{\mathrm{ab} *}$, Simon D. M. Jacques ${ }^{\mathrm{abc} *}$, Marco Di Michiel ${ }^{\mathrm{d}}$, Vesna Middelkoop ${ }^{\mathrm{e}}$, \\ Christopher K. Eganc, Robert J. Cernik ${ }^{\mathrm{c}}$ and Andrew M. Beale ${ }^{\text {ab* }}$ \\ aDepartment of Chemistry, University College London, 20 Gordon Street, London, WC1H 0AJ, UK \\ bUK Catalysis Hub, Research Complex at Harwell, Didcot, Oxfordshire \\ 'School of Materials, University of Manchester, Manchester, Lancashire, M13 9PL, UK \\ ${ }^{\mathrm{d}}$ ESRF - The European Synchrotron, Grenoble, F-38000, France \\ ${ }^{e}$ Flemish Institute for Tecnological Research, VITO NV, Boeretang 200, Mol, Belgium
}

Correspondence email: antonyvam@gmail.com;simon.jacques@gmail.com; andrew.beale@ucl.ac.uk

\section{XRD-CT method}

A schematic representation of an XRD-CT experiment is provided in Figure S1. The method relies on a pencil beam scanning approach using a highly collimated or focussed monochromatic beam with, for best counting statistics/speed, scattered X-rays recorded on an area detector. This is typically normal to and centred with respect to the beam. Typically, the object is translated across the X-ray beam (i.e. perpendicular to the beam axis) with a step size close/same as the horizontal size of the incoming X-ray beam and for every translational step $t$, diffraction patterns are collected using an area detector. The length of the translational scan can be the same as the width of the sample but in practise the value for the length $s$ used is higher (i.e. number of translational steps $n=s / t$ ). This is because one should take into account the imperfection of the sample alignment and also the fact that the sample may move during successive tomographic collections. After a translational scan is completed, the sample is rotated (angular step) and the translational scan is repeated. The angular range usually covered is from 0 to $\pi$ and the number of angles scanned $m$ should be, as in the case of the first generation X-ray computed tomography, equal to the number of translational measurements times $\pi / 2$ (i.e. $m=n \times \pi / 2$ ). However, in practice, the number of angular steps can be decreased without significant changes in the quality of the collected data (Álvarez-Murga et al., 2012). 


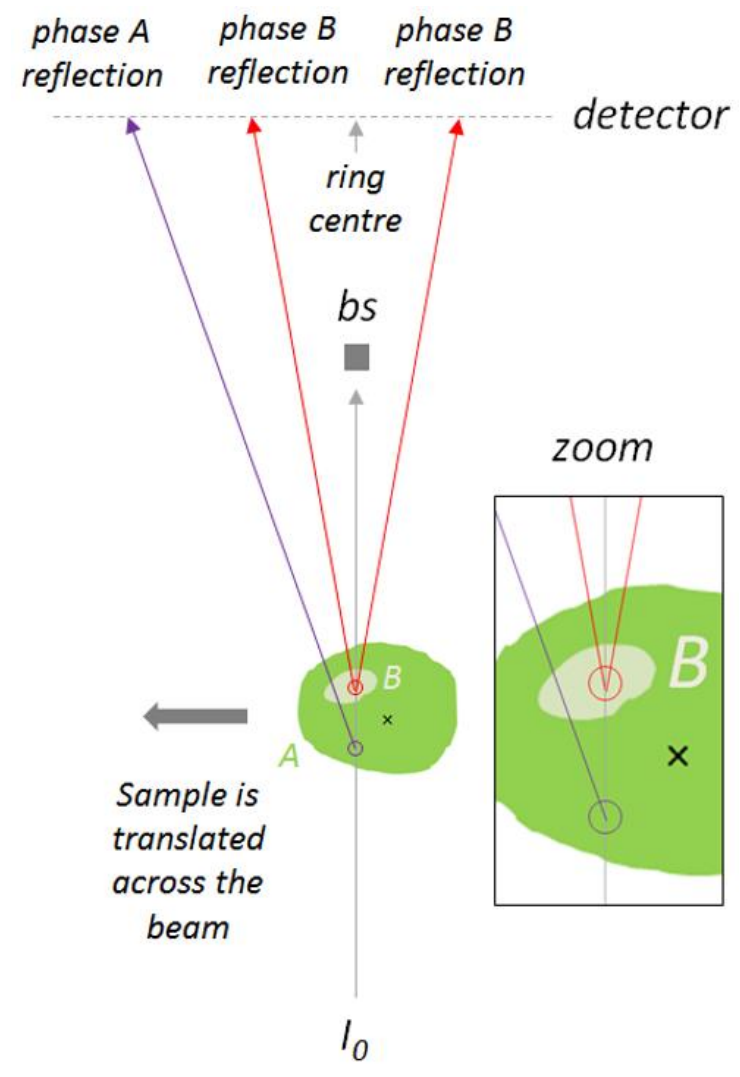

Figure S1: Tomographic plane schematics of XRD-CT experiment. In the schematic the green object consists primarily of phases A (coloured green) with inclusion of a small amount of phase B (coloured light grey/green). The incident beam is denoted $\mathrm{I}_{0}$, the detector is protected from the incident beam by use of a backstop (denoted bs in the schematics). As the object is translated across the beam, diffraction is recorded from the entire path of the incident beam. If A and B are powders, characteristic Bragg reflections from both phases will be recorded on the detector manifesting as diffraction rings on the detector. This is illustrated in the figure where purple ray path indicates a reflection at high $2 \theta$ angles from phase A, and the red paths indicate a single reflection from phase $\mathrm{B}$ recorded at low $2 \theta$ angles on different sides of the detector ring centre. 


\section{Preparation of catalyst and membranes}

$2 \% \mathrm{Mn}-1.6 \% \mathrm{Na}-3.1 \% \mathrm{~W} / \mathrm{SiO}_{2}$ was prepared by a sequential incipient wetness impregnation method. Firstly, the $\mathrm{SiO}_{2}$ support (Silica gel Davisil 646, 250-500 $\mu \mathrm{m}$ ) was impregnated by an aqueous solution of sodium tungstate dihydrate $\mathrm{Na}_{2} \mathrm{WO}_{4} \cdot 2 \mathrm{H}_{2} \mathrm{O}$ and sodium oxalate $\mathrm{Na}_{2} \mathrm{C}_{2} \mathrm{O}_{4}$ salts taken in appropriate concentrations at a temperature of $80^{\circ} \mathrm{C}$. The $\mathrm{Na}-\mathrm{W} / \mathrm{SiO}_{2}$ was dried at $120{ }^{\circ} \mathrm{C}$ for $6 \mathrm{~h}$ and was then impregnated by an aqueous solution of manganese (II) acetate tetrahydrate $\mathrm{Mn}(\mathrm{CH} 3 \mathrm{COO})_{2} \cdot 4 \mathrm{H}_{2} \mathrm{O}$ salt. For the $2 \% \mathrm{La}-2 \% \mathrm{Mn}-1.6 \% \mathrm{Na}$ $3.1 \% \mathrm{~W} / \mathrm{SiO}_{2}$ catalyst, the $\mathrm{Na}-\mathrm{W} / \mathrm{SiO}_{2}$ was dried at $120^{\circ} \mathrm{C}$ for $6 \mathrm{~h}$ and was then impregnated by an aqueous solution of manganese (II) acetate tetrahydrate $\mathrm{Mn}(\mathrm{CH} 3 \mathrm{COO})_{2} \cdot 4 \mathrm{H}_{2} \mathrm{O}$ and lanthanum nitrate $\mathrm{La}\left(\mathrm{NO}_{3}\right)_{3} \cdot 6 \mathrm{H}_{2} \mathrm{O}$ salts taken in appropriate concentrations. The catalysts were then dried at $120^{\circ} \mathrm{C}$ for $6 \mathrm{~h}$ and calcined in air at 850 for $6 \mathrm{~h}$ with a heating rate of $2^{\circ} \mathrm{C} / \mathrm{min}$. The BCFZ membranes reported here have been manufactured using the spinning and phase inversion methods previously described.(Van Noyen et al., 2012, Middelkoop et al., 2014) The starting polymer suspension was prepared from cellulose acetate (CA, Mr 52000, Fluka), dimethylsulphoxide (DMSO, Synthesis grade, Merck) and de-ionised water that were used as a phase-inversion polymer, solvent and non-solvent additive to the polymer solution, respectively. 


\section{Summed diffraction pattern}

The summed diffraction pattern of the XRD-CT dataset is shown at the left side of Figure S2. The cristobalite (green), $\mathrm{BaWO}_{4}$ (red), $\mathrm{Mn}_{2} \mathrm{O}_{3}$ (cyan) and $\mathrm{BCFZ}$ (magenta) peaks presented at the right side of Figure S2 correspond to the sinograms used in this work.
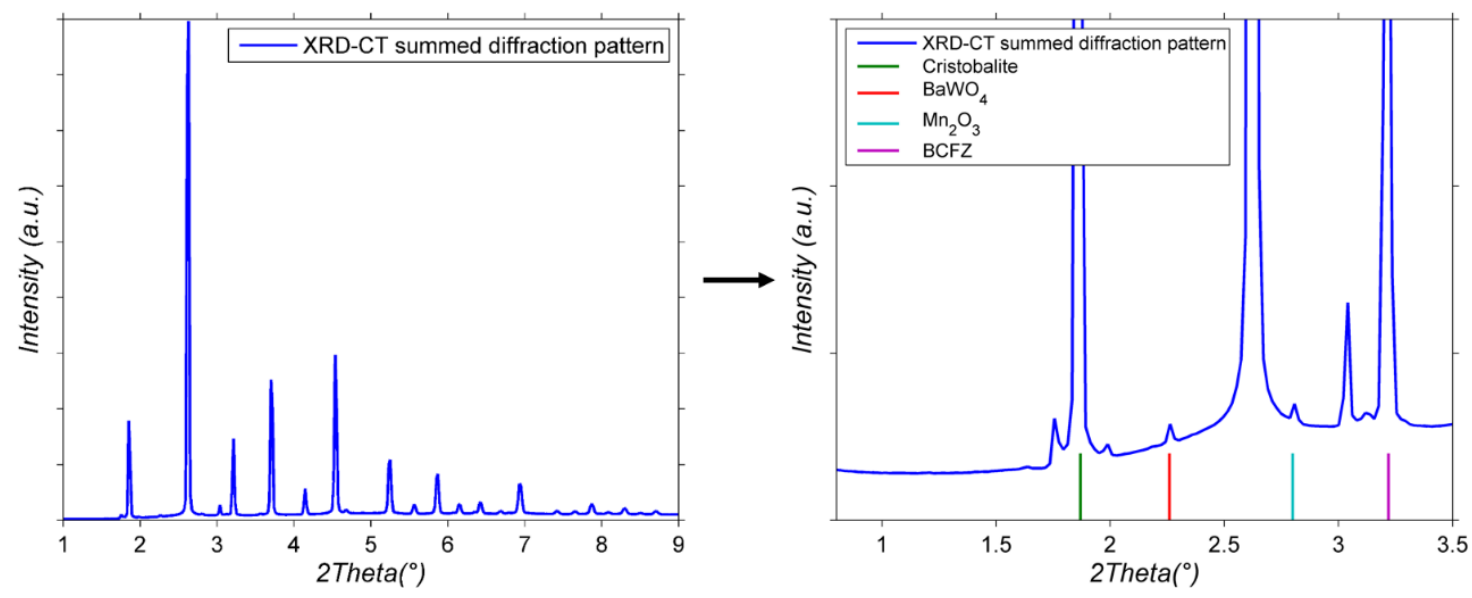

Figure S2: Left: The summed diffraction pattern from the XRD-CT dataset presented in the main paper. Right: A region of interest (ROI) of that diffraction pattern, showing four peaks corresponding to cristobalite (green), $\mathrm{BaWO}_{4}(\mathrm{red}), \mathrm{Mn}_{2} \mathrm{O}_{3}(\mathrm{cyan}$ ) and BCFZ (magenta) phases.

\section{Tomographic reconstruction algorithms}

The effect of different reconstruction algorithms to deal with spotty sinograms is presented in Figure S3. The sinogram used corresponds to $\mathrm{BaWO}_{4}$ also shown in Figure 3 in the main paper. The XRDUA software program was used to implement the various tomographic algorithms. Initially, the $\mathrm{CeO}_{2}$ (NIST) diffraction pattern was used for calibration and then the XRD-CT data were processed (i.e. azimuthal integration of the raw 2D diffraction images and creation of the sinograms). As expected (see first row in Figure S8), the $\mathrm{BaWO}_{4}$ sinogram and reconstructed XRD-CT image (i.e. using the filtered back projection algorithm) are identical with the ones generated using the MATLAB script used in this work. Different tomographic algorithms were implemented to reconstruct the spotty BaWO4 sinogram using the XRDUA software. More specifically, the algebraic reconstruction technique (ART), the simultaneous algebraic reconstruction technique (SART), the simultaneous iterative reconstruction technique (SIRT), the ordered subset expectation minimization (OSEM) and the maximum likelihood expectation maximization (MLEM) algorithms were tested. As it is shown in Figure S8, the reconstructed images are full of line artefacts regardless of the algorithm used (or how many iterations are used). 

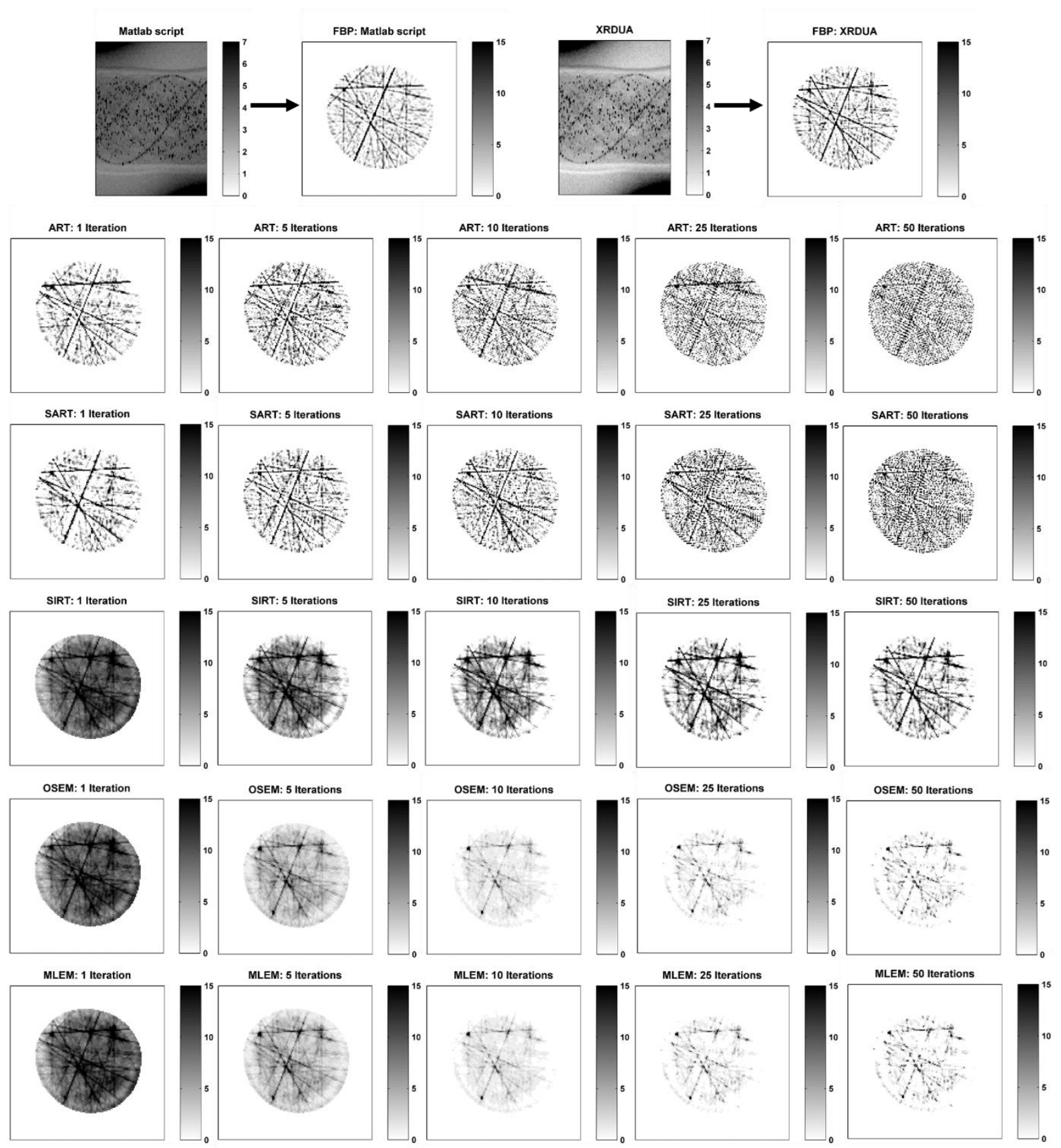

Figure S3: Top row: $\mathrm{BaWO}_{4}$ sinograms and reconstructed images (FBP) using the MATLAB script provided in the ESI and the XRDUA software program. The reconstructed images when ART, SART, SIRT, OSEM, MLEM are used is also shown. 


\section{Alpha-trimmed mean filter}

In Figure S4, the derived 1D diffraction patterns for the $\mathrm{CeO}_{2}$ standard using different values for the alpha-trimmed mean are shown (i.e. $0,1,2,3,5,10,25,50$ and $75 \%$ respectively). The 2D diffraction image shown in Panel A of Figure 2 in the main text is used as the benchmark tool. As it is shown in Figure S4, there are no obvious changes in the 1D diffraction patterns using the different filters.

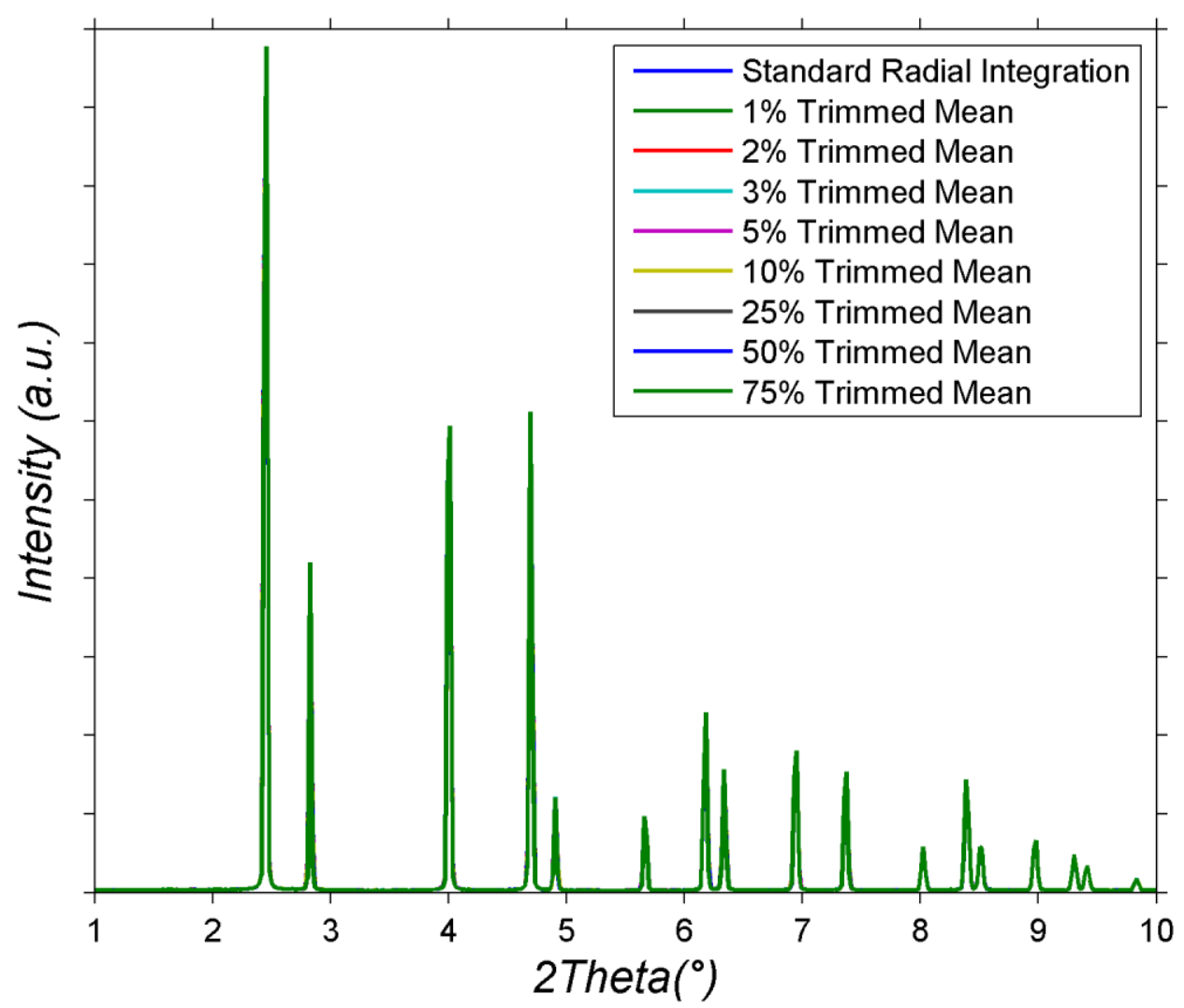

Figure S4: The 1D diffraction patterns for the $\mathrm{CeO}_{2}$ standard are shown using different values for the alpha-trimmed mean filter.

In Figure S5, the relative difference between the 1D diffraction pattern calculated with standard radial integration and 1,2 and 3\% alpha-trimmed mean filter is presented. It can be seen that the values remain less than $10 \%$ even for the $3 \%$ alpha-trimmed mean filter. 


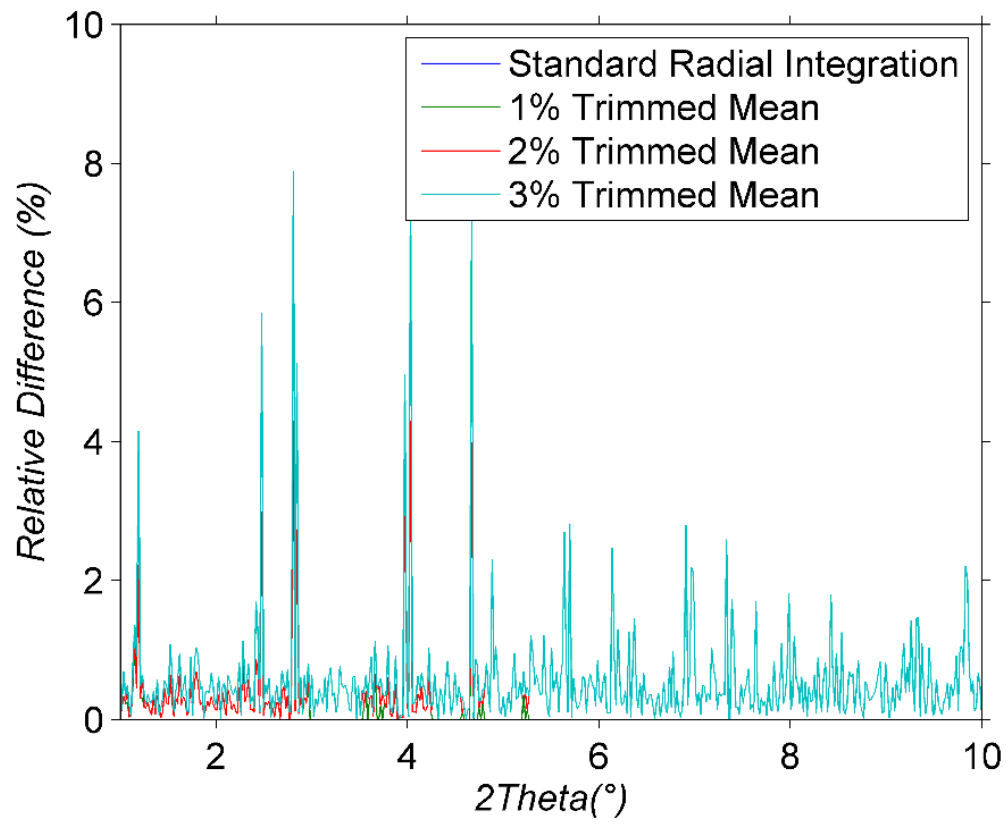

Figure S5: The relative difference between the 1D diffraction pattern calculated with standard radial integration and 1, 2 and $3 \%$ alpha-trimmed mean filter for the $\mathrm{CeO}_{2}$ is shown.

In Figure S6, the relative difference between the 1D diffraction pattern calculated with standard radial integration and 10,25, 50 and $75 \%$ alpha-trimmed mean filter is presented. As expected, the values increase and there are differences more than $50 \%$ for the $75 \%$ alpha-trimmed mean filter at certain scattering angles $2 \theta$.

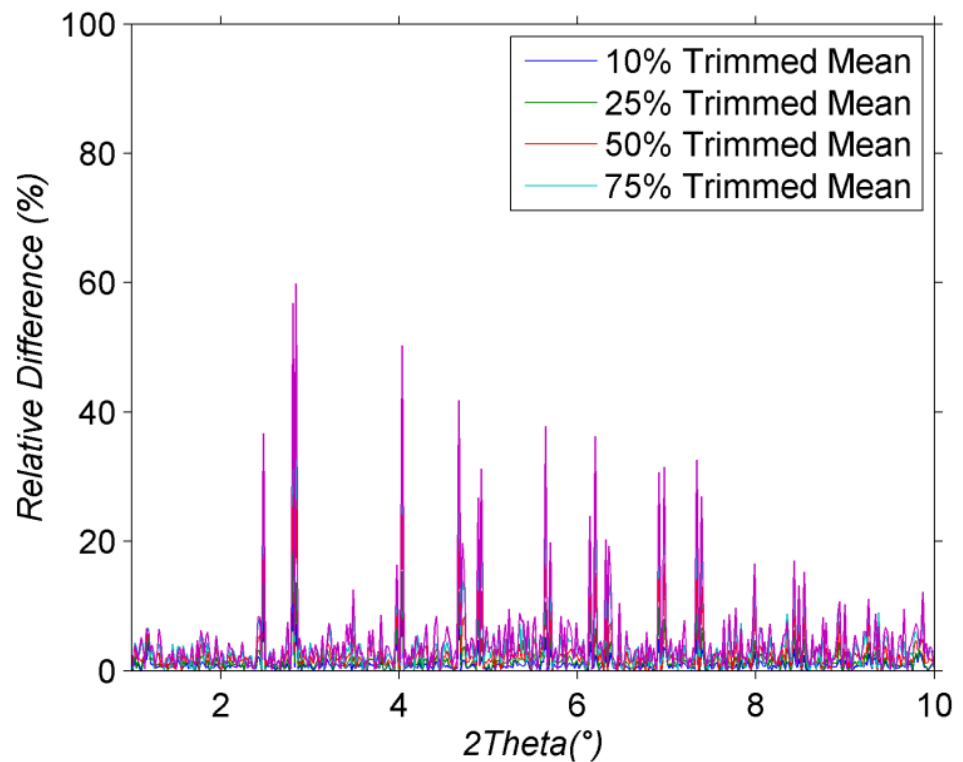

Figure S6: The relative difference between the 1D diffraction pattern calculated with standard radial integration and 1, 2 and $3 \%$ alpha-trimmed mean filter for the $\mathrm{CeO}_{2}$ is shown 
The maximum values of the relative difference between the 1D diffraction pattern calculated with standard radial integration and 1,2,3, 5, 10, 25, 50 and $75 \%$ alpha-trimmed mean filter for the $\mathrm{CeO} 2$ are presented in Figure S7.

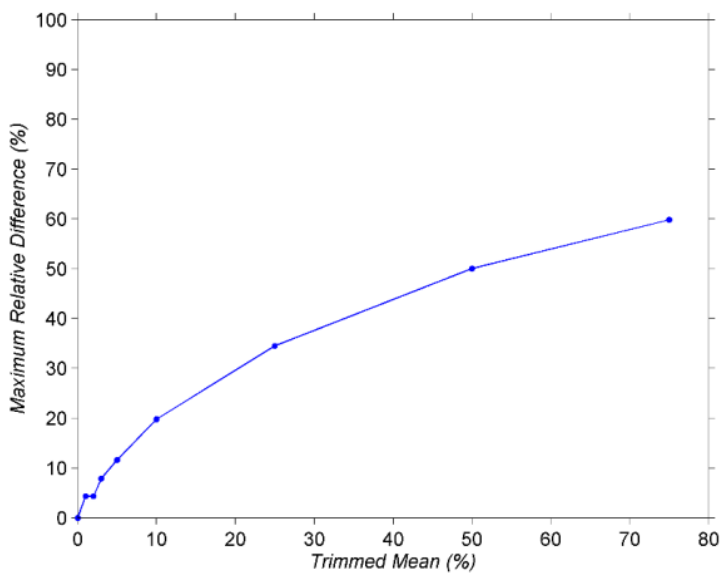

Figure S7: The maximum values of the relative difference between the 1D diffraction pattern calculated with standard radial integration and 1,2, 3, 5, 10, 25, 50 and $75 \%$ alpha-trimmed mean filter for the $\mathrm{CeO}_{2}$ is shown.

However, as it was shown also in Figure S4, even for the extreme case of the $75 \%$ trimmed mean filter, the changes are not radical. The maximum intensities of the diffraction peaks are maintained and the main changes (e.g. the 60\% reported in Figure S5) are observed at the tails of the bragg peaks. This is clearly shown in Figure S8 where the 1D diffraction pattern calculated with standard radial integration and the one calculated with $75 \%$ trimmed mean filter are presented. 


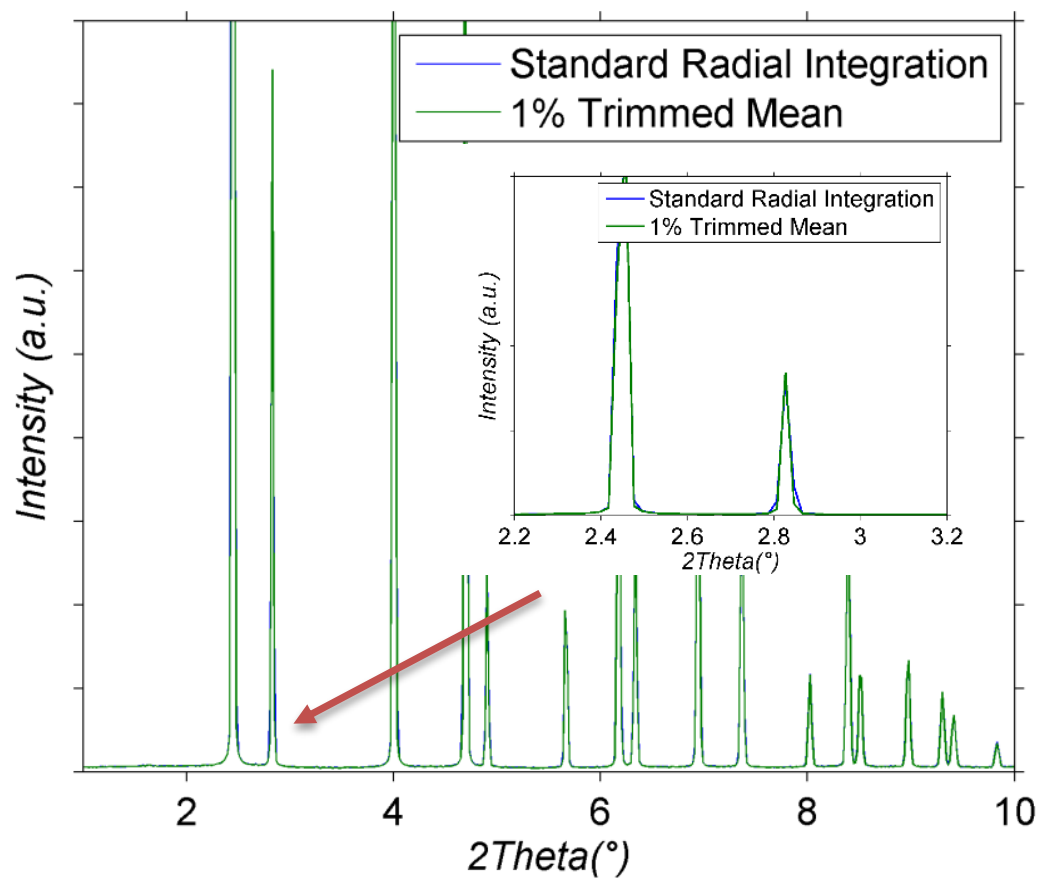

Figure S8: The 1D diffraction patterns for the $\mathrm{CeO}_{2}$ standard is shown using standard radial integration and 75\% alpha-trimmed mean filter. Also shown is a $2 \theta$ region of interest where the maximum relative difference between the two diffraction patterns is observed (i.e. scattering angle $2.85^{\circ} 2 \theta$. 


\section{MATLAB Scripts}

The main MATLAB in-house function used in this work are provided below.

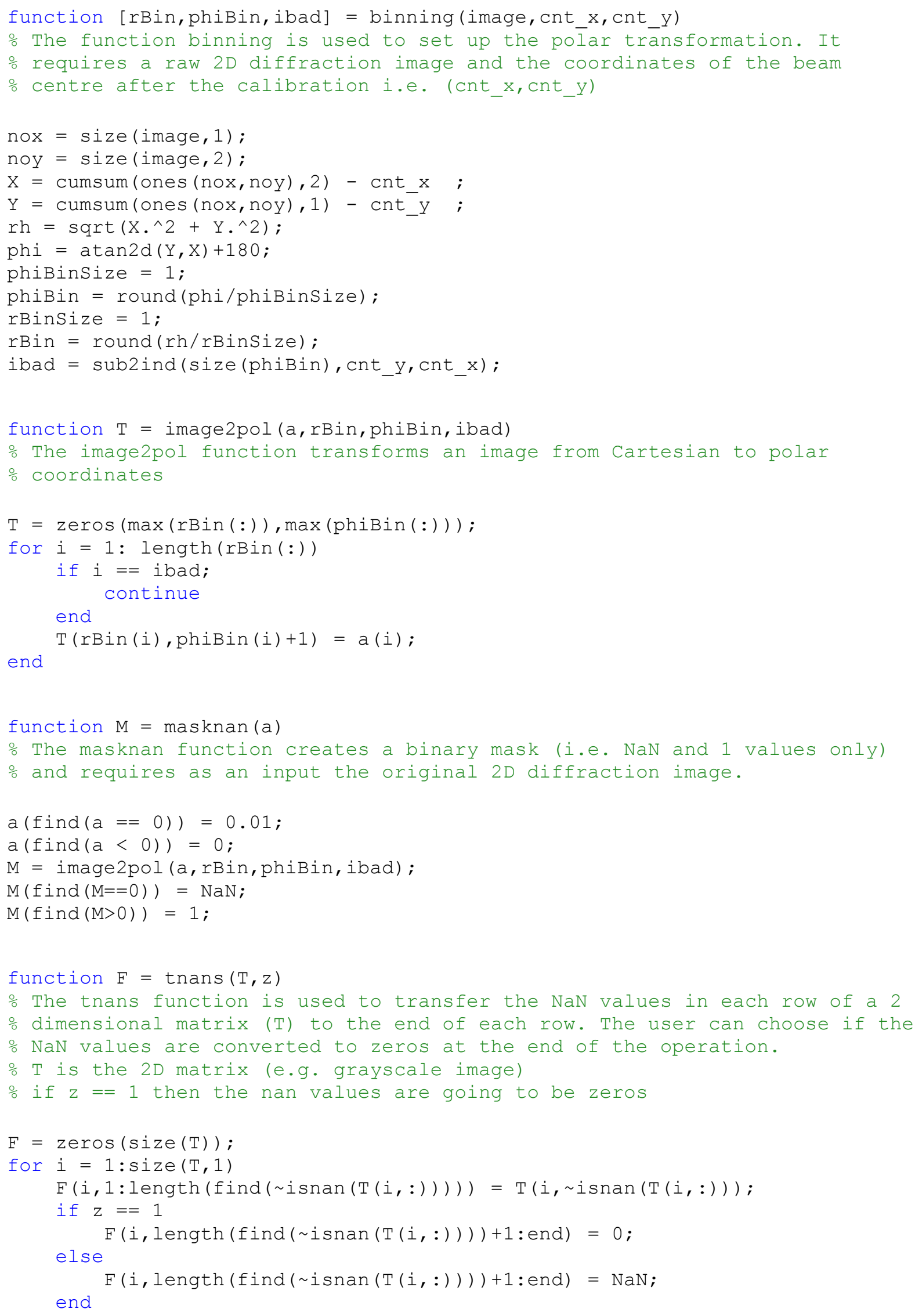




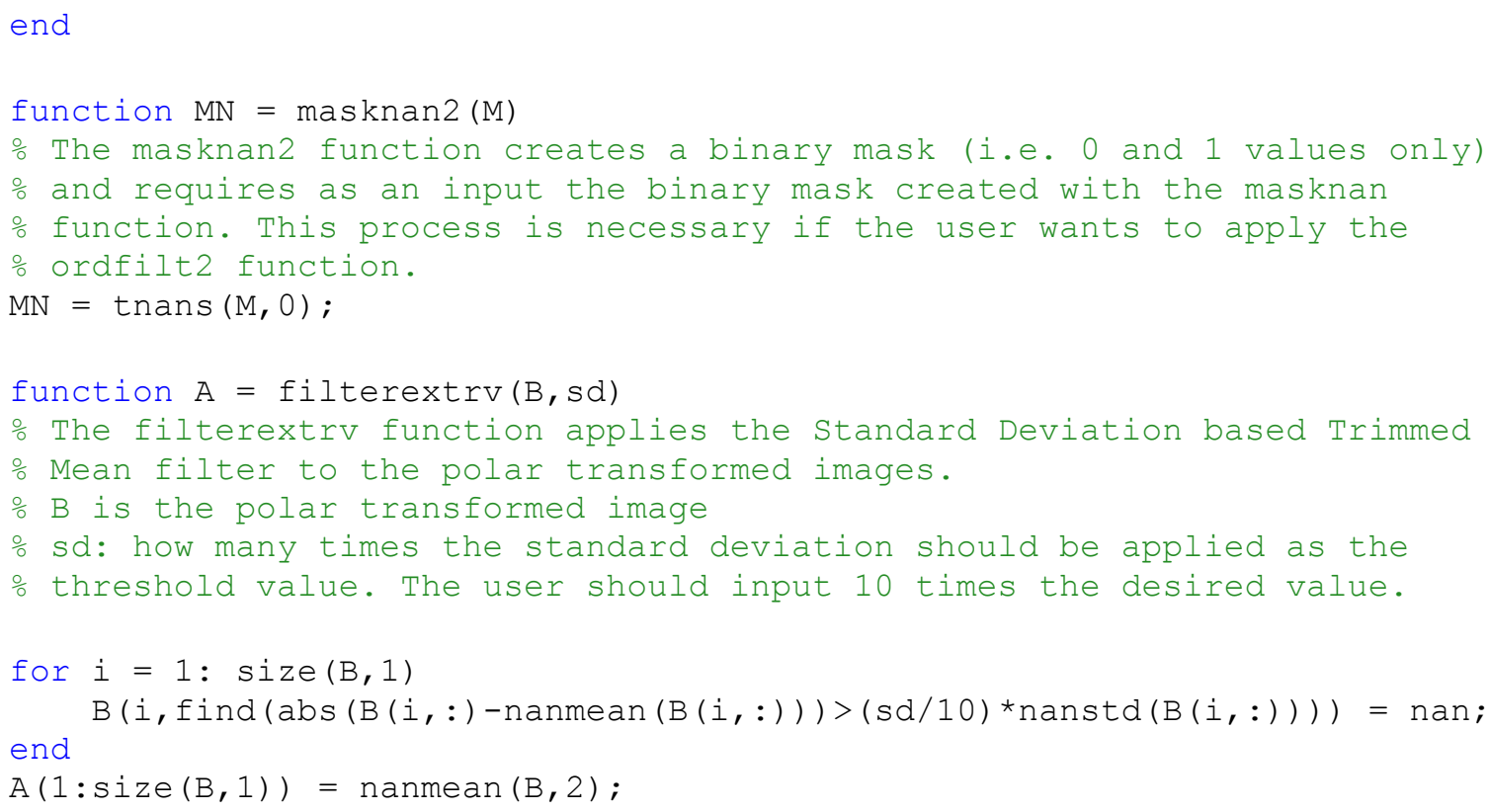

Below is provided, for the interested reader, a simplified example of the MATLAB code used to apply different filter in the XRD-CT data presented in this work.

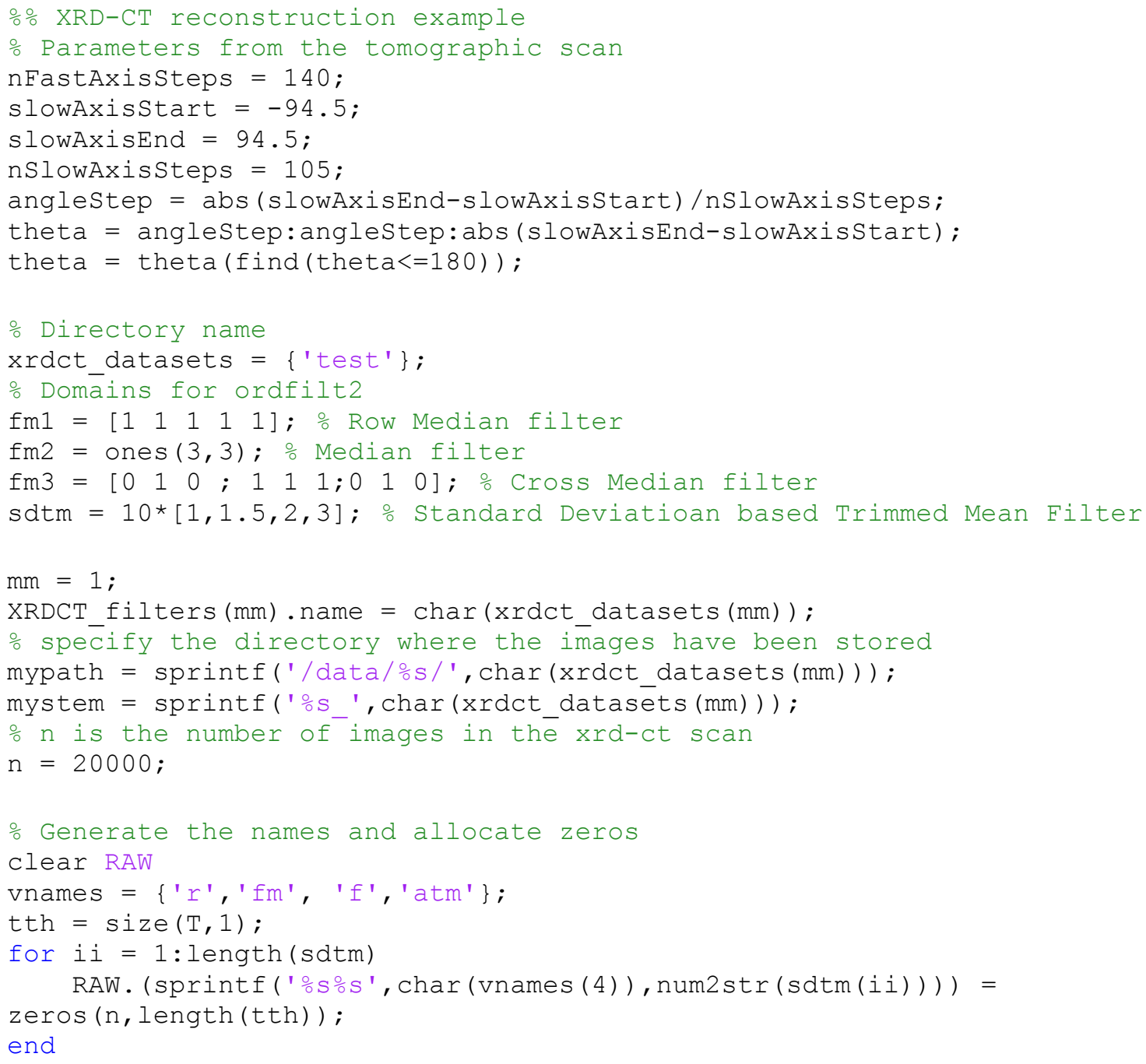




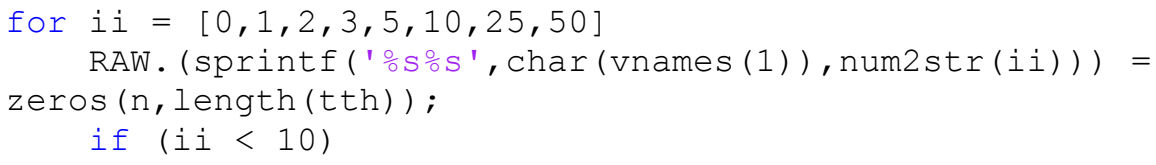

RAW. (sprintf ('ㅇsㅇs 1 ', char (vnames (1)), num2str(ii), char (vnames (2)))) (jj+1, : ) = trimmean (F1 (1: length (tth), : ), i , 2) ' ;

RAW. (sprintf ('ㅇsㅇs 2 ', char (vnames (1)), num2str(ii), char (vnames (2)))) (jj+1, : ) = trimmean (F2 (1: length (tth), : ), ii,2)' ;

RAW. (sprintf ('응ㅇs 3 ', char (vnames (1)), num2str(ii), char (vnames (2)))) (jj+1, : ) = trimmean (F3 (1: length (tth), : ), ii,2)' ; end

end end

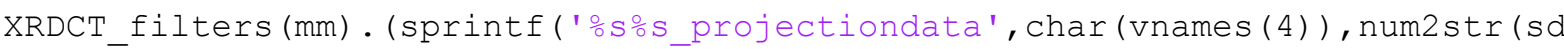
$\operatorname{tm}(i \bar{i})))=$

reshape (RAW. (sprintf ('ㅇs', char(vnames (4)), num2str(sdtm(ii)))), nFastAxisSt eps, nSlowAxissteps+1, size $(T, 1))$;

end

for ii $=[0,1,2,3,5,10,25,50]$ 
XRDCT_filters (mm). (sprintf('\%s\%s_projectiondata', char(vnames (1)), num2str(ii )) ) $=$ reshape (RAW. (sprintf ('응ㅇ's , char (vnames (1)), num2str(ii))), nFastAxisSteps, nS lowAxisteps+1, size $(\mathrm{T}, 1))$;

if $(i i<10)$

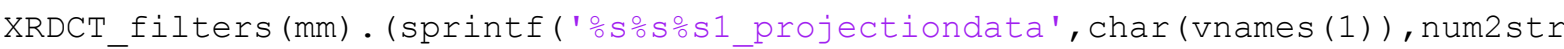
(ii), $\overline{c h a r}(\operatorname{vnames}(2)))$ ) =

reshape (RAW. (sprintf ('sss 1', char (vnames (1)), num2str(ii), char (vnames (2))) ), nFastAxissteps, nSlowAxisSteps+1, size (T,1));

XRDCT_filters (mm). (sprintf('\%s\%s\%s2_projectiondata', char(vnames (1)), num2str (ii), $\operatorname{char}(\operatorname{vnames}(2)))$ ) =

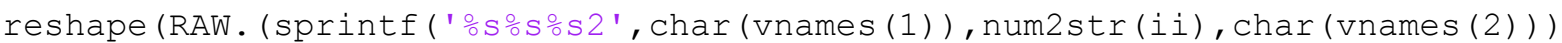
), nFastAxissteps, nSlowAxisSteps+1, size (T,1));

XRDCT_filters (mm). (sprintf('ㅇsㅇs 3_projectiondata', char (vnames (1)), num2str (ii), $\operatorname{char}(\operatorname{vnames}(2)))$ ) =

reshape (RAW. (sprintf ('sss 3', char (vnames (1)), num2str(ii), char (vnames (2))) ), nFastAxissteps, nSlowAxisSteps+1, size (T,1)); end

end

\% Centre the sinograms

for $i \mathrm{i}=1: 1:$ length $(\mathrm{sdtm})$

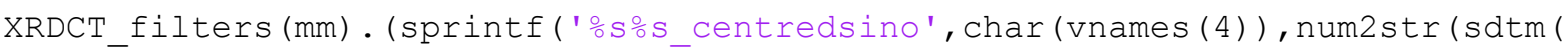
ii)) ) $\bar{l}=$

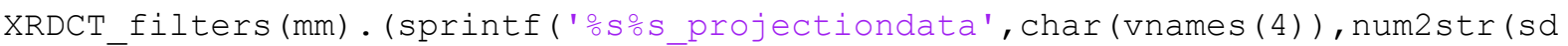
$\operatorname{tm}(i \bar{i})))(:, 1:$ length (theta), : );

end

for $i \mathrm{i}=[0,1,2,3,5,10,25,50]$

XRDCT_filters (mm).(sprintf('\%s\%s_centredsino', char(vnames (1)), num2str(ii))) $=$

XRDCT_filters (mm).(sprintf('으요rojectiondata', char(vnames (1)), num2str(ii ))) ( : , $1:$ length (theta), : );

if $(i i<10)$

XRDCT_filters (mm). (sprintf('ㅇsㅇs__centredsino', char (vnames (1)), num2str(ii ), $\operatorname{char}(\operatorname{vnames}(2))))=$

XRDCT_filters (mm). (sprintf('\%s\%s\%s1_projectiondata', char(vnames (1)), num2str (ii), $\operatorname{char}(\operatorname{vnames}(2)))$ ) (:, 1: length (theta), :);

XRDCT_filters (mm). (sprintf('\%s\%s\%s2_centredsino', char(vnames (1)), num2str (ii ), $\operatorname{char}(\operatorname{vnames}(2)))$ ) =

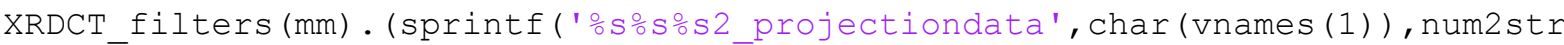
(ii), $\operatorname{char}(\operatorname{vnames}(2)))$ ) (:, 1: length (theta), :);

XRDCT filters (mm). (sprintf('ㅇsㅇs 3 centredsino', char (vnames (1)), num2str(ii ), $\operatorname{cha} \bar{r}(\operatorname{vnames}(2))))=$

XRDCT_filters (mm). (sprintf('\%s\%s\%s3_projectiondata', char(vnames (1)), num2str

(ii), $\operatorname{char}(\operatorname{vnames}(2)))$ ) (:, 1: length (theta), :) ; end

end

\% Filtered back projection

$i \mathrm{i}=0$; 


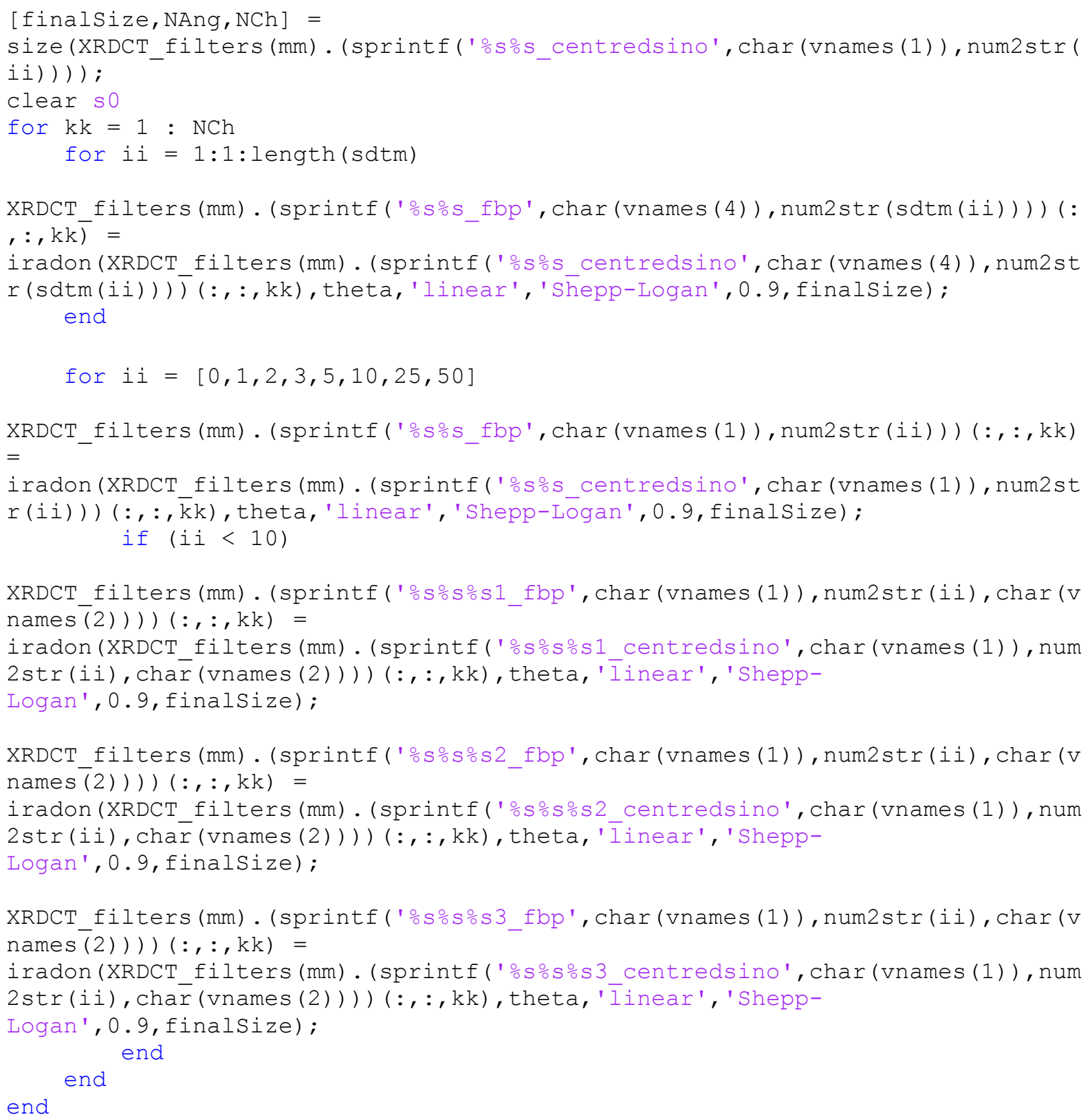




\section{References}

Middelkoop, V., Chen, H., Michielsen, B., Jacobs, M., Syvertsen-Wiig, G., Mertens, M., Buekenhoudt, A. \& Snijkers, F. (2014). J. Membr. Sci. 468, 250-258.

Van Noyen, J., Middelkoop, V., Buysse, C., Kovalevsky, A., Snijkers, F., Buekenhoudt, A., Mullens, S., Luyten, J., Kretzschmar, J. \& Lenaerts, S. (2012). Catal. Today 193, 172-178. 